\title{
Re-using Prior Tactile Experience by Robotic Hands to Discriminate In-Hand Objects via Texture Properties
}

\author{
Mohsen Kaboli ${ }^{1}$, Rich Walker ${ }^{2}$, and Gordon Cheng ${ }^{1}$
}

\begin{abstract}
This paper proposes an online tactile transfer learning strategy for discriminating objects through the surface texture properties via a robotic hand and an artificial robotic skin. The proposed method has the ability to autonomously select and exploit the previously learned multiple texture models while discriminating new textures with a very few available training samples or even one. The experimental results show that employing the proposed method and 10 prior texture models, the robotic hand could discriminate 12 objects via their surface textures with $97 \%$ and $100 \%$ recognition accuracy with only one and ten training samples respectively. Moreover, the experimental outcomes illustrate that our proposed algorithm is robust against of any negative tactile knowledge transfer.
\end{abstract}

\section{INTRODUCTION}

\section{A. Motivation}

Tactile information is pivotal for autonomous robots for detecting and learning the physical properties of objects. The performance of tactile systems depends not only on the technological aspect of the sensory device [1] but also on the design of the learning methods that interpret information contained in tactile data [2].

\section{B. Background}

Liu et al. employed an intelligent contact sensing finger to classify surface materials with Naive Bayes classifier [3]. $\mathrm{Hu}$ et al. used Support Vector Machine (SVM) to classify five different fabrics by sliding a finger-shaped sensor over the surfaces [4]. A robot actively knocks on the surface of the experimental objects with an accelerometer-equipped device to discriminate stone, mulch, moss, and grass from each other with a lookup table and k-nearest neighbors (K-NN) techniques [5]. To classify cotton, linen, silk, and denim fabrics, Song et al. designed a mechanism to generate the relative motion at a certain speed between the PVDF film and surface of the perceived fabric. In this study neural network and K-means clustering algorithms were used for fabric surface texture recognition [6]. A force sensor, an accelerometer, and a position-orientation sensor were used to develop a haptic tool which was then used by a robotic hand in order to identify surface textures through multiple One-Class Support Vector Machines [7]. Five textiles were explored and discriminated from each other via $\mathrm{k}$-nearest neighbor (k-NN) using an active sliding touch strategy and an array of MEMS [8]. A humanoid robot was equipped with an artificial finger nail with

\footnotetext{
${ }^{1}$ Institute for Cognitive Systems, Faculty of Electrical Engineering and Information Technology, Technical University of Munich (TUM)- Germany. Email: mohsen.kaboli@tum.de (http://www.ics.ei.tum.de)

${ }^{2}$ Shadow Robot Company-UK.
}

\section{Prior Objects}

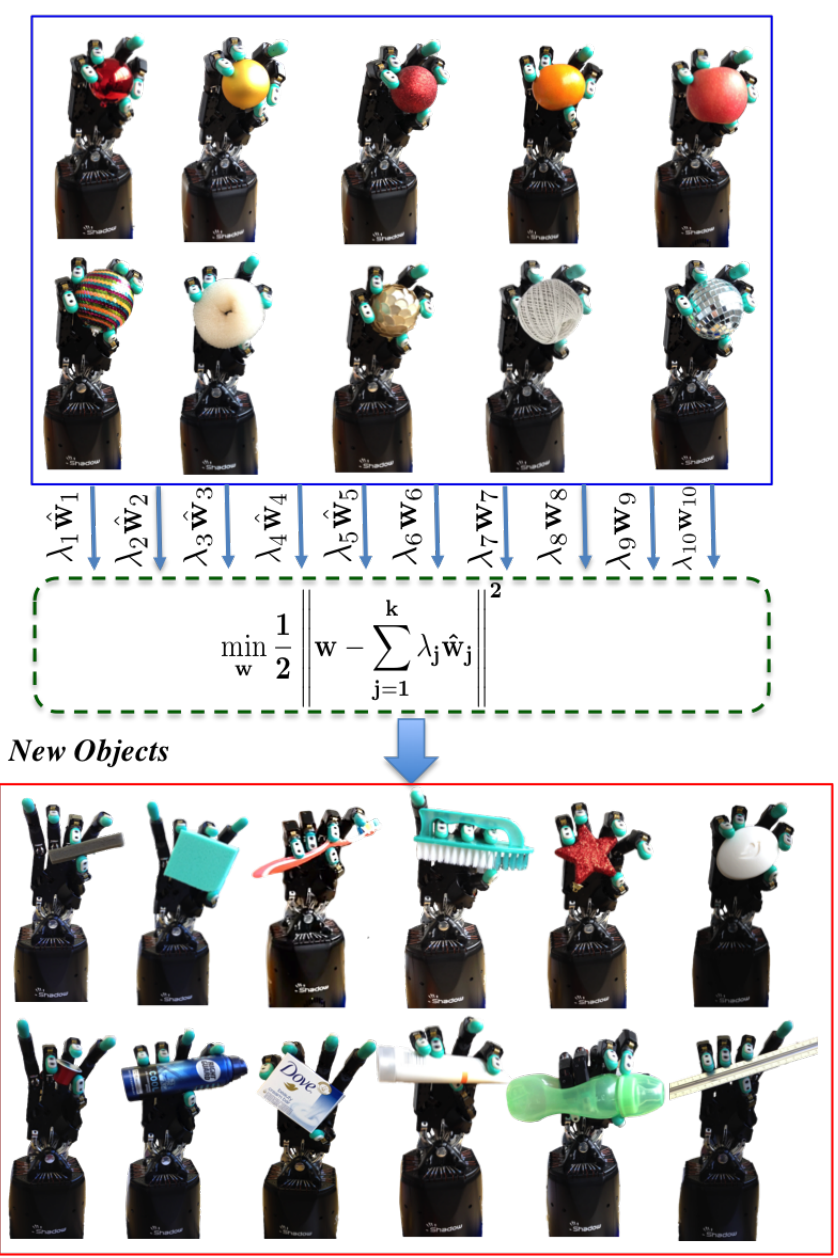

Fig. 1. The Shadow Hand with BioTac robotic skin. Employing the proposed tactile transfer learning method the Shadow Hand could re-use its prior knowledge while discriminating new objects from their texture with a very few trials. The illustrated formula is the regularizer term of the adapted LS-SVM in (2). The weight assigned to each prior knowledge $\lambda$ was found by minimizing Eq.(2). $K$ is the number of prior knowledge.

an attached 3-axis accelerometer in order to classify 20 different surfaces through Support Vector Machine (SVM) and k-nearest neighbor (k-NN) learning techniques [9]. Jamali et al. fabricated a biologically inspired artificial finger composed of silicon with two PVDF pressure sensors and two strain gauges. The finger was mounted on a robotic gripper and was scraped over eight materials. The Majority voting learning method was employed to find the optimal technique for the texture recognition problem 
[10]. Classification results of 117 different textures using Bayesian exploration are reported in [11]. A multi-modal tactile sensor called BioTac was used to perceive tactile information. In this experiment one BioTac sensor was placed on a customized tool and a vibration-free linear staged was used to slide textures under the tactile sensor. In [12], the Shadow Hand with the BioTac sensor on the index fingertip together with Bayesian exploration technique were employed to discriminate 10 different objects while executing exploratory movements over the surface of the objects. However, the existing texture classification methods cannot re-use the past tactile experience or prior learned texture models (tactile transfer learning). There are many papers proposing various transfer learning strategies in other robotic fields than tactile sensing [13]-[17]. However, these proposed methods are not appropriate for tactile learning tasks due to the properties of the tactile signals and tactile features. In our previous research, we have shown that it is necessary to re-design appropriate feature descriptors and learning methods for tactile data processing and modeling specially for surface texture discrimination [18], [19]. This is especially important when a large area of a robotic system covered with many tactile sensors [20].

\section{Contribution}

The aim of this study is to enable robotic hands to re-use the already learned object texture models while classifying new in-hand objects from their textures with a few available training samples or even one, especially in an online manner. In this respect we propose, for the first time in tactile learning area, a novel online tactile transfer learning technique.

\section{SYSTEM DESCRIPTION}

\section{A. Robotic Hand}

The Shadow Hand is a dexterous Robotic Hand System with five fingers and 20 active degrees of freedom in total, which enables the robot to have a range of movement equivalent to that of a human hand (see Fig.2).

\section{B. Multi-Modal Artificial Skin}

The BioTac is a multi-modal tactile sensor designed to provide the ability to replicate the human sense of touch. When the sensor moves over an object, caused vibration can be measured to obtain a dynamic pressure signal $\left(\mathbf{P}_{A C}\right)$ with the sampling data rate of $2 \mathrm{KHz}$. The BioTac also has 19 impedance-sensing electrodes $\left(\mathbf{E}_{1}, \ldots, \mathbf{E}_{19}\right)$ distributed over the surface of the rigid part. These electrodes are capable of measuring the deformation that arises when normal forces are applied to the surface of the skin with a $50 \mathrm{~Hz}$ sampling data rate (see Fig.2).

\section{Properties Of Experimental Objects}

In this work 22 everyday objects (natural and synthetic) were selected. 10 objects were chosen with an identical geometrical shape property (spherical shape), including a Red and a Yellow ball with almost similar smooth surface

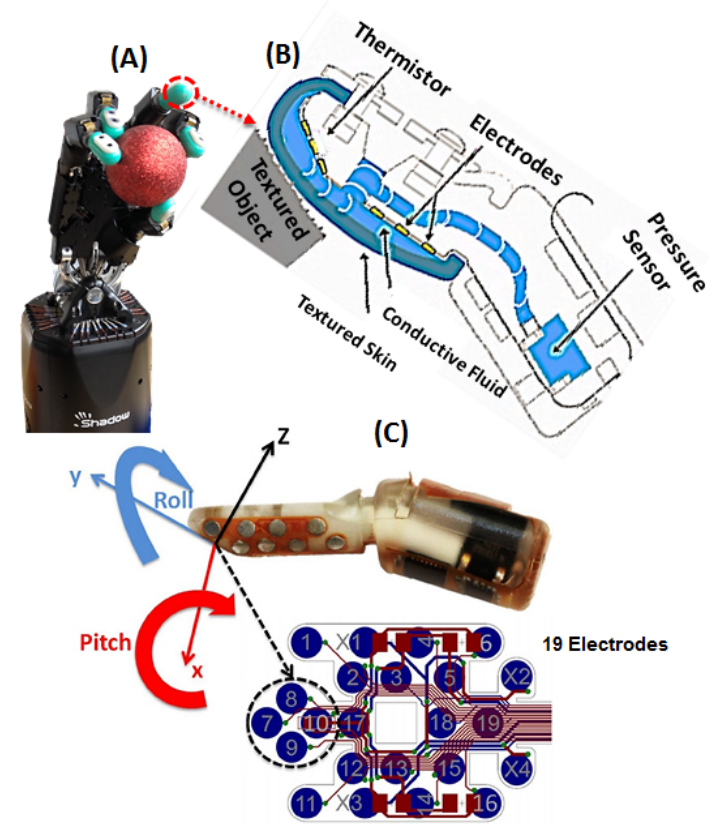

Fig. 2. Fig.A shows the Shadow Robotic Hand, Fig.B shows the schematic of the BioTac sensor while sliding on a textures surface, and Fig.C shows the BioTac sensor with 19 impedance electrodes.

texture, a Rough textured ball, an Orange, an Apple, a Colorful ball with smooth and non-uniform texture, a Rough spherical sponge, a Pine apple textured ball (non-uniform texture), a String ball, and a Mirror ball (see Fig.1-Prior Objects). Also, 12 objects with different shapes including a Soft sponge, a Memory sponge (non-uniform texture), a Toothbrush (non-uniform texture), a Floor brush, a Rough textured star (non-uniform texture), a Soap, a Spray, a Coffee capsule, a Paper box, a Cream tube, a Plastic baby feeder, and a Metal ruler (see Fig.1-New Objects). The first set of the objects was used to construct a prior tactile knowledge and the second set was considered as new objects which the robotic hand should recognize them with the help of its prior tactile knowledge. In both sets of the objects, the difference in the surface texture properties between the selected objects varied from relatively similar to noticeably different.

\section{Tactile Perception And Data Collection}

\section{A. Data Collection With Prior Objects Set}

The Shadow Hand held each of the spherical shaped prior objects (see Fig.1-Prior Objects) in palm with three random fingers. Afterwards, the robotic hand explored the texture of each in-hand object by randomly moving the remaining two free fingers to slide over the surface of the in-hand object for 3 seconds. The texture exploration was repeated 50 times for each prior objects with random orientation. The entire collected data (for each object) then randomly divided in two sets, one set for the training purpose with 30 samples and the other set with 20 trials for the testing. 


\section{B. Data Collection With New Objects Set}

In this scenario, the robotic hand used its three fingers to hold each of the complex shaped object (Fig.1-New Objects). The surface exploration carried out with the remaining two fingers by sliding over the surface of each object for 3 seconds. The data collection was repeated 30 times for each new object. The entire collected data (for each new object) was divided in two sets, 10 samples for the training and 20 for the testing.

\section{Feature Extraction Methodology}

Previously, we proposed a novel set of tactile feature descriptors for texture discrimination task [19]. In this study, we employed our proposed parameters to extract robust feature from the measured tactile data during object texture exploration.

\section{Tactile Transfer Learning Methodology}

\section{A. Motivation and problem definition}

Consider a scenario in which the Shadow Hand has already constructed a set of tactile learning models to discriminate $k=10$ different surface textures (see Fig.1Prior Objects) with sufficient enough available training samples. Now the task of the Shadow Hand is to classify $M=12$ new surface textures (see Fig.1-New Objects) with only one or a very few available training samples while re-using the previous learned texture models ( Prior texture models) in an online manner. Our proposed hybrid tactile transfer learning method has four main steps. 1Constructing Prior Texture Models 2- Autonomously selecting the most relevant multiple texture models for the new texture recognition (Prior Texture Model Selection) 3.1- Initializing the online learning algorithm with the constructed prior models 3.2- Constructing the new texture models while receiving new textures/objects 4- Updating And Re-weighting the Prior Textures Models. The initial idea comes from our method in [16], [21] which we re-designed and extensively improved it for the tactile data.

\section{B. Constructing Prior Texture Models}

The Shadow Hand employed the Least Squared Support Vector Machine (LS-SVM) [22] to construct several prior texture models. In this case the LS-SVM was trained with $k=10$ prior textures (see Fig. 1-Prior Objects). More formally, consider a classification scenario with an entire available set of training data $\left\{\mathbf{x}_{\ell}, y\right\}_{\ell=1}^{N_{k}}$ where $\ell=1, \ldots, N_{k}$, $k=10$ is the number of prior objects/textures, $N_{k}$ is a number of training samples of each prior object, $\mathbb{X} \subset \mathbb{R}^{d}$ is an input vector describing the $\ell^{\text {th }}$ sample and $y \in \mathbb{Y}$ is the corresponding objects' label. The main purpose is to construct a function, $g_{j}(\mathbf{x})=\hat{\mathbf{w}}_{j} \cdot \mathbf{x}(j=1, \ldots, k)$ that can divide the unseen test data. In this respect, $\phi(\mathbf{x})$ is utilized to map the input trial samples to a higher dimensional feature space, in our case, radial basis kernel. In LS-SVM the texture model parameters $(\mathbf{w}, b)$ are obtained by solving

$$
\min _{\mathbf{w}, b} \frac{1}{2}\|\mathbf{w}\|^{2}+\frac{C}{2} \sum_{\ell=1}^{N}\left[y-\mathbf{w} \cdot \phi\left(\mathbf{x}_{\ell}\right)-b\right]^{2} .
$$

where $C$ is a regularization parameter that controls the bias-variance trade-off. $N$ is the number of the training samples collected with each prior objects (in our case $N=30)$.

\section{Prior Texture Model Selection}

By slightly modifying the regularization term in LS-SVM (1), it is possible to construct new discriminating texture models for the new objects (see Fig.1-New Objects) close to the already constructed prior models (see Fig.1-Prior Objects):

$$
\min _{\mathbf{w}, b} \frac{1}{2}\left\|\mathbf{w}-\sum_{j=1}^{k} \lambda_{j} \hat{\mathbf{w}}_{j}\right\|^{2}+\frac{C}{2} \sum_{\ell=1}^{N}\left[y-\mathbf{w} \cdot \phi\left(\mathbf{x}_{\ell}\right)-b\right]^{2} .
$$

where $\hat{\mathbf{w}}$ is the parameter describing the prior texture models and $\lambda$ is a scaling factor corresponding to ranking the prior models and decides how much and from where to transfer the prior tactile knowledge. In other words $\lambda$ controls to what degree the new texture models are close to the prior texture models. The optimization problem (2) has the same cost function as LS-SVM in which the regularizer term has been modified to impose closeness between the new texture models and a linear combination of prior texture models. The weight factor $\lambda$ assigned to each prior texture model was found by minimizing $\sum_{t=1}^{k} \ell_{t}(\tilde{y}, y)$ subject to $\|\boldsymbol{\lambda}\|_{2} \leq 1$ where $\tilde{y}$ is the leave one out prediction for the $t-t h$ sample and $\lambda=\left(\lambda_{1}, \ldots, \lambda_{k}\right)$. With this formulation the final prediction function for the collected testing data is:

$$
g(\mathbf{x})=\mathbf{w} \cdot \mathbf{x}+b=\left(\sum_{j=1}^{k} \lambda_{j} \hat{\mathbf{w}}_{j}+\sum_{t=1}^{T} \alpha_{t} \mathbf{x}_{t}\right) \cdot \mathbf{x}+b .
$$

In (3) $\alpha_{t}$ are the coefficients of the support vectors for the new textures classification problem.

\section{On-line Learning Algorithm (PA)}

Passive Aggressive (PA) is an open-ended classification technique [23]. PA algorithm constructs a classification model continuously at the time of receiving new samples. More formally, PA estimates the model parameter $\mathbf{w}_{t}$ at every time $t$ receives new data samples and accordingly predicts the corresponding labels. At $t=1$, the PA starts with $\mathbf{w}_{1}=(0, \ldots, 0) \in \mathbb{R}^{d}$, then PA updates the constructed models while receiving new samples by solving (4).

$$
\mathbf{w}_{t+1}=\min _{\mathbf{w} \in \mathbf{R}^{d}} \frac{1}{2}\left\|\mathbf{w}-\mathbf{w}_{t}\right\|^{2}+\eta \xi,
$$

which results in Eq.(4).

$$
\mathbf{w}_{t+1}=\mathbf{w}_{t}+\theta_{t} \mathbf{y}_{t} \mathbf{x}_{t}
$$


where

$$
\theta_{t}=\min \left\{\eta, \frac{\max \left\{0,1-\mathbf{y} \mathbf{w}_{t} \cdot \mathbf{x}_{t}\right\}}{\left\|\mathbf{x}_{\mathbf{t}}\right\|^{2}}\right\}
$$

In (4), $\eta$ is a positive value that governs the influence of the slack terms. In (5), $\mathbf{x}_{t}$ is the current received sample at time $t$ and $\mathbf{y}_{t}$ is the label of the received sample.

\section{E. Online Tactile Transfer Learning}

This is a hybrid algorithm in which the adapted LSSVM provides autonomously the most relevant prior models $k=10$ to the new texture models $M=12$. This results in constructing an initial new texture models:

$$
\mathbf{w}_{1}=\left(\sum_{s=1}^{k} \lambda_{s} \hat{\mathbf{w}}_{s}+\sum_{t=1}^{T} \alpha_{i} \mathbf{x}_{i}\right) .
$$

The (7) is composed of two parts. The first part is the linear combination of the weighted prior texture models where $\mathbf{w}_{s}$ is the prior model, $\lambda_{s}$ is the scaling factor (needs to be updated at each time $t$ ), and $k$ is the number of prior models. The second part represents the received new training texture samples ( $T$ is the number of the samples). Now, the PA algorithm uses the new initial models $\mathbf{w}_{1}$ in (7) instead of the $\mathbf{w}_{1}=(0, \ldots, 0)$ to learn from the $(t+1)-t h$ new incoming texture samples.

Updating And Re-weighting the Prior Textures Models: So far, we initialized the PA learning algorithm by integrating the prior and new texture models. But, still, the prior texture models are not directly re-weighted during the on-line learning process. We describe here how the weights of the prior and new texture models will be updated during the on-line learning progressively in time. In this case, the prediction can be made on each new incoming samples by means of the current constructed texture models in (7) as $\mathbf{w}_{1} \cdot \mathbf{x}_{t}$.

The results of the prediction $\sigma_{k, t}$ will be cropped between $(-1,1)$ and will be used as the $(d+k)-t h$ element $(d$ is the dimension of a new sample and $k$ is the number of the prior models) in the feature vector of $\mathbf{x}_{t}$ defined as:

$$
\mathbf{x}_{t}^{\prime}=\left(\mathbf{x}_{t}, \sigma_{1, t}, \ldots, \sigma_{k, t}\right) \in \mathbb{R}^{d+k}
$$

where

$$
\sigma_{k, t}=\max \left\{-1, \min \left\{1, \mathbf{w}_{1}^{k} \cdot \mathbf{x}_{t}\right\}\right\} .
$$

The new samples with such a modified representation enters the online algorithm. At $t=1$ online algorithm predicts with $\operatorname{sign}\left(\mathbf{w}_{1}^{\prime} \cdot \mathbf{x}_{1}^{\prime}\right)$ in which the $\mathbf{w}^{\prime}{ }_{1}=\left(\mathbf{w}_{1}, \mathbf{1}\right) \in \mathbb{R}^{d+k}$. For the $t+1$ the updating rule in Eq.(5) now is

$$
\mathbf{w}_{t+1}^{\prime}=\mathbf{w}_{t}^{\prime}+\theta_{t} y_{t} \mathbf{x}_{t}^{\prime}
$$

where

$$
\theta_{t}=\min \left\{C, \frac{\max \left\{0,1-y \mathbf{w}_{t}^{\prime} \cdot \mathbf{x}_{t}^{\prime}\right\}}{\left\|\mathbf{x}_{t}^{\prime}\right\|^{2}}\right\} .
$$

and the final predictions are

$$
\mathbf{w}_{t}^{\prime} \cdot \mathbf{x}_{t}^{\prime}=\sum_{i=1}^{t-1} \theta_{i} y_{i}(\underbrace{\mathbf{x}_{i} \cdot \mathbf{x}_{t}}_{\text {New Samples }}+\underbrace{\sigma_{k, i} \sigma_{k, t}}_{\text {Prior knowledge }}) .
$$

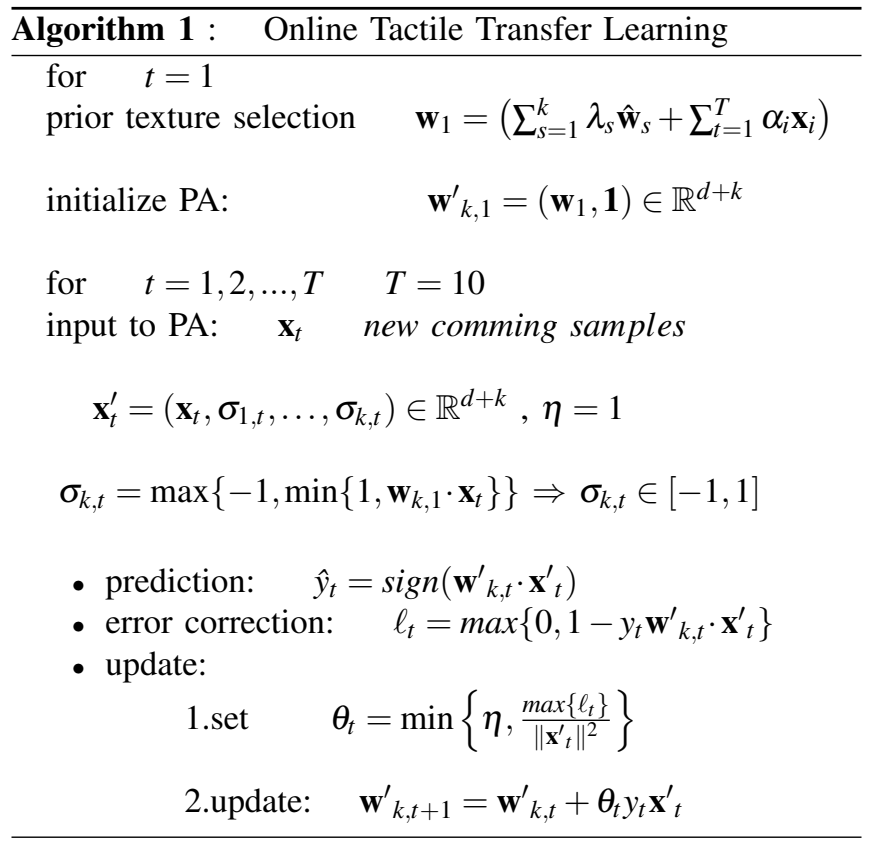

Hence $\mathbf{w}_{t}^{\prime}$ is composed of two parts, one part is the knowledge coming from the new instances in sequence and the other part is the prior texture knowledge (see Algorithm 1).

\section{EXPERIMENTAL RESUlts}

In this section we show empirically the effectiveness and consistency of our proposed online tactile transfer algorithm.

\section{A. Constructing Prior Tactile Models}

In order to construct 10 prior texture models LS-SVM classifier was employed. The entire training samples (30 training samples for each prior texture) were split in two parts, $70 \%$ for training and $30 \%$ for the testing. Five-fold cross validation was applied to find the optimal kernel parameter and regularizer value $C$. LS-SVM was then re-trained with the entire collected training data and the obtained optimal parameters to construct 10 prior texture models. The learned texture models $(\mathbf{w}, \mathbf{b}) \in R^{10}$ were then evaluated by predicting on unseen collected test data (20 test samples for each class of prior texture). The Shadow Hand using LSSVM could classify successfully 10 prior textures with $100 \%$ recognition accuracy.

\section{B. Evaluating The Proposed Online Transfer Learning}

The Shadow Hand used the proposed tactile transfer learning technique to recognize 12 new objects via their textures. The proposed algorithm enabled the Shadow Hand to re-use $k=10$ already constructed prior texture models while learning from a very few new training samples. In this scenario, the new textures/objects (see Fig.1-New Objects) entered to the proposed hybrid online transfer learning sequentially one after one to construct new hybrid learning models. At each time $t=1, \ldots, 10$, the constructed leaning models were evaluated by predicting on unseen new test data. The prediction results were reported as a recognition rate in Fig. 3-(A). 


\section{Base Line}

In order to compare our proposed method with the traditional online learning method, the traditional PA algorithm was employed to construct surface texture models while receiving new training samples continuously over time (one new texture per time $(t=1, \cdots, 10)$. The new constructed learning models at each time $t$ were evaluated by predicting on unseen new test data (20 test samples per new textures). The classification results were reported as a recognition rate in Fig. 3-(A). The value for $\eta$ was fixed to 1 in both hybrid online transfer learning and PA online leaning (base line). Fig. 3-(A) shows that using our proposed hybrid online transfer learning method the Shadow Hand could discriminate 12 new textures with $97 \%$ recognition accuracy while using only one new training sample plus ten prior models. By increasing the number of training samples from one to ten, the Shadow Hand achieved $100 \%$ recognition accuracy. The results in Fig. 3-(A) illustrates that our proposed method outperforms the traditional online learning.

The computational cost of the online tactile transfer learning approach is $\mathscr{O}\left(T^{2}+N^{3}+k N^{2}\right)$. In other words, the computational complexity of our proposed algorithm is the sum of the computational cost of the PA algorithm $\left(\mathscr{O}\left(T^{2}\right)\right)$ and adapted LS-SVM $\left(\mathscr{O}\left(N^{3}\right)+\mathscr{O}\left(k N^{3}\right)\right) . T$ is the total number of new textures, $k$ is the number of the prior texture models, and $N$ is the number of the trials used to construct the hybrid leaning models with adapted LS-SVM. In our proposed method only a few number of trials entered to the adapted LS-SVM in order to construct the learning models (7). Hence, the term $\left(\mathscr{O}\left(N^{3}\right)+\mathscr{O}\left(k N^{3}\right)\right)$ in the proposed method is negligible. Therefore, our proposed method and PA online algorithm have similar computational complexity. It is worth to mention that growing the number of prior textures increases the probability to find more useful and related prior models for the new textures. Moreover, it is important to mention that our proposed method will have substantially higher performance if the texture properties of the prior and new objects will be more similar to each other.

\section{Decreasing The Number Of Prior Tactile Knowledge}

In this scenario 5 out of 10 prior objects randomly selected to construct the new prior models. All procedure explained in V-A, V-B, V-C was reaped with randomly selected 5 prior objects. Moreover, in order to evaluate the robustness of our proposed tactile learning algorithm, the experiment was repeated 50 more times. Fig. 3-(B) illustrates the averaged recognition rate over 50 experiments. The results in Fig. 3(B) shows that our proposed method outperforms the traditional online learning. In this scenario, the Shadow Hand achieved $94 \%$ and almost $100 \%$ recognition accuracy with only one and ten trials, respectively. Although having more prior knowledge increases the chance to find more relevant prior information for new tasks (therefore, higher recognition rate), it increases the computational complexity of the transfer learning algorithm. For instance the computation time of our algorithm with 10 and 5 prior knowledge was $360 \mathrm{~ms}$ and $330 \mathrm{~ms}$, respectively (PC with Intel(R) Core(TM) i7-4510U
CPU@2.00 GHz 2.60 GHz 32 GB Ram). This becomes more serious when the number of prior knowledge will increase to 100,000 or $1,000,000$. Solving such a constrain in any transfer learning approach can be a new challenge to tackle for the future research.

\section{E. Negative Knowledge Transfer Consistency Experiment}

In transfer learning scenario the constructed prior models are not always relevant to new object/texture models. If the prior models are dissimilar to the new models, brute force transfer can degrade the recognition performance, generating so called negative knowledge transfer. Ideally, a transfer learning method should be beneficial between prior and new models while avoiding negative transfer when the object surface textures are not a good match. We show that our proposed tactile transfer learning technique is robust against of the negative knowledge transfer. In this respect, Expectation Maximization algorithm was employed to find out which of the new textures are similar or dissimilar to the prior textures. In this case, the EM was trained with entire training samples (10 samples per each texture) to cluster 20 objects (both prior and new object textures). The EM then was evaluated by unseen test data (20 samples per each texture). Fig. 4 illustrates the resulted confusion matrix. Regarding to the obtained confusion matrix, Spray, Metal ruler, Pine apple, and String ball did not have any similarity with the prior object in terms of surface texture properties (see Fig. 1-Prior Objects). In this scenario, Spray, Metal ruler, Pine apple, and String ball were selected as a set of new textures while the prior textures were remain same. The hybrid online transfer learning was employed to discriminate the four new textures and traditional PA was used as a base line. The rest of the procedure was similar to $\mathrm{V}-\mathrm{B}$.

Fig. 3-(C) illustrates the classification results in terms of recognition accuracy. The results clearly show that the obtained recognition performance while using the proposed hybrid online transfer learning is similar to the performance achieved while using the traditional PA. This means that our algorithm stopped transferring irrelevant prior knowledge to new task.

\section{Conclusion}

In this study we focused on tactile knowledge transfer across texture categories. We proposed an online tactile transfer learning method to provide the robotic systems with the ability of re-using previously learned tactile models (prior models) to discriminate new textures with a very few available training samples.

In this study, the distributions of the tactile information in both prior knowledge and new tasks were similar. In future we will solve the problem in which the data distributions (feature space) in both prior and new data are different. It is also interesting interesting if it will be possible to transfer tactile information to new tasks in which prior and new tactile data are measured by different types of tactile sensors (different in characteristic and technology). 


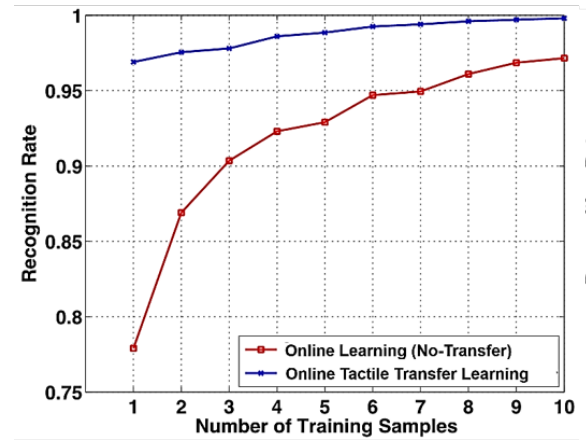

(A)

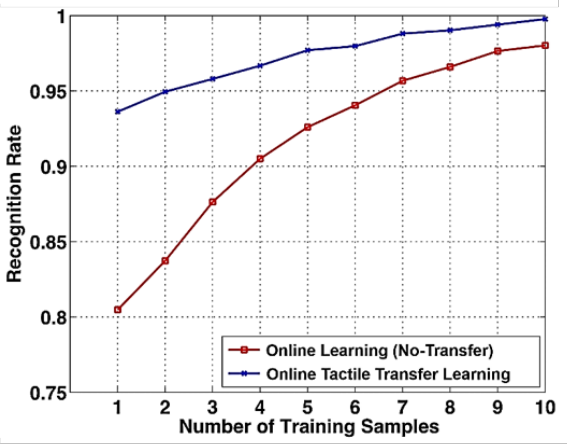

(B)

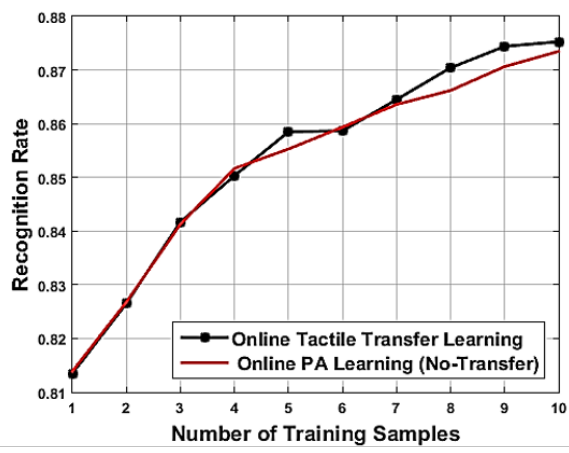

(C)

Fig. 3. Figure (A) and (B) show the recognition results on a separate test data for the online tactile transfer learning and traditional PA online learning (No-Transfer) methods. In these experiments 10 and 5 prior texture models were re-used in (A) and (B) respectively by the Shadow Hand. Figure (C) shows the recognition results corresponding to the hybrid tactile transfer learning and traditional transfer learning (No-Transfer) in which the new surface textures were dissimilar to the prior textures. The recognition results on the new test set were plotted as a function of the number of the training samples

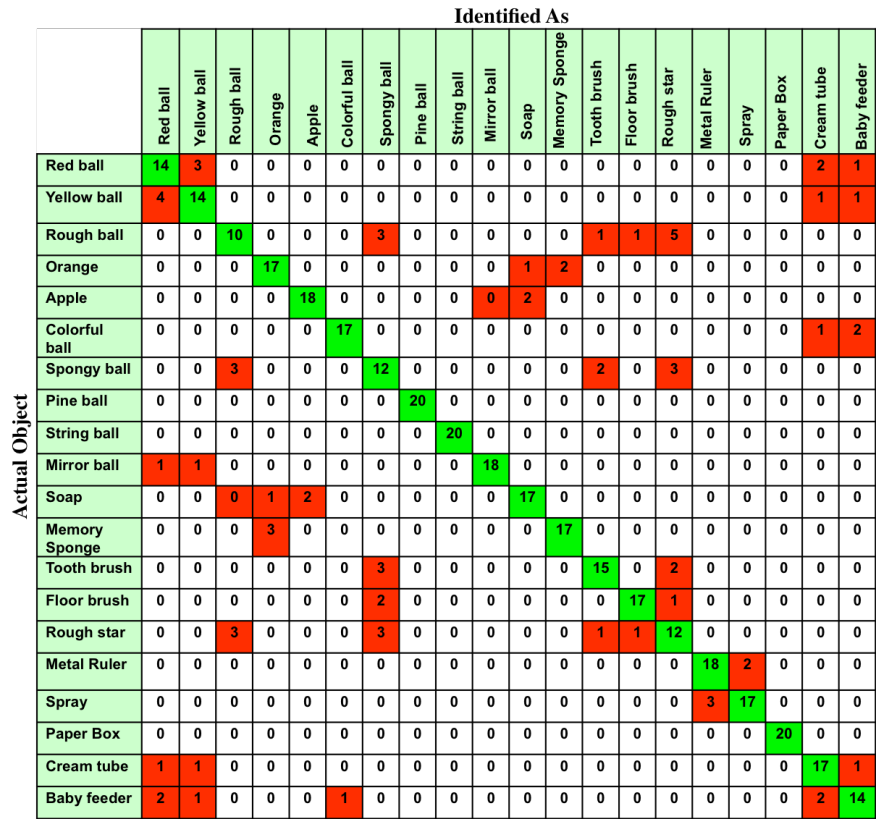

Fig. 4. This figure shows the confusion matrix for the clustering of 20 objects via texture properties using EM method.

\section{ACKNOWLEDGMENT}

This work is supported by the European Commission under grant agreements PITN-GA-2012-317488-CONTEST.

\section{REFERENCES}

[1] N. Yogeswaran, et al., "New materials and advances in making electronic skin for interactive robots," Advanced Robotics, vol. 29, no. 21, pp. 1359-1373, 2015.

[2] R. Dahiya, P. Mittendorfer, M. Valle, G. Cheng, and V. Lumelsky, "Directions towards effective utilization of tactile skin-a review," IEEE Trans. on Robotics, 2013.

[3] H. Liu, X. Song, J. Bimbo, L. Seneviratne, and K. Althoefer, "Surface material recognition through haptic exploration using an intelligent contact sensing finger," IEEE Int. Conf. on Intelligent Robots and Systems, pp. 152-57, 2012.

[4] H. Hu, Y. Han, A. Song, S. Chen, C. Wang, and Z. Wang, "A fingershaped tactile sensor for fabric surfaces evaluation by 2-dimensional active sliding touch," Sensors, vol. 14, pp. 4899-4913, 2014.
[5] J. Windau and W. Shen, "An inertia-based surface identification system," IEEE Int. Conf. on Rob. and Aut., pp. 2330-2335, 2010.

[6] A. Song, Y. Han, H. Hu, and J. Li, "A novel texture sensor for fabric texture measurement and classification," IEEE Trans. on Instrumentation and Measurement, vol. 63, no. 7, pp. 1739-1747, 2014.

[7] J. M. Romano and K. J. Kuchenbecker, "Methods for robotic toolmediated haptic surface recognition," IEEE Haptics Symposium, pp. 49-56, 2014.

[8] C. M. Oddo, M. Controzzi, L. Beccai, C. Cipriani, and M. C. Carrozza, "Roughness encoding for discrimination of surfaces in artificial activetouch," IEEE Trans. on Robotics, vol. 27, pp. 522-533, 2011.

[9] J. Sinapov, V. Sukhoy, R. Sahai, and A. Stoytchev, "Vibrotactile recognition and categorization of surfaces by a humanoid robot," IEEE Trans. on Robotics, vol. 27, no. 3, pp. 488-497, 2011.

[10] N. Jamali and C. Sammut, "Majority voting: Material classification by tactile sensing using surface texture," IEEE Trans. on Robotics, vol. 27, pp. 508-521, June 2011.

[11] A. J. Fishel and G. E. Loeb, "Bayesian exploration for intelligent identification of textures," Front. in Neurorob., vol. 6, no. 4, 2012.

[12] D. Xu, G. E. Loeb, and A. J. Fishel, "Tactile identification of objects using bayesian exploration," in IEEE Int. Conf. on Robotics and Automation, pp. 3056-3061, May 2013.

[13] Y. Zhu, Y. Chen, Z. Lu, S. J. Pan, and Q. Y. G.R. Xue, Y. Yu, "Heterogeneous transfer learning for image classification," Proceedings of the Twenty-Fifth AAAI Conf. on Artificial Intelligence, 2011.

[14] P. Zhao and C. H. Steven, "Ott: A framework of online transfer learning.," Int. Conf. on Machine Learning, 2010.

[15] S. J. Pan and Q. Yang, "A survey on transfer learning," IEEE Trans. on Knowledge and Data Engineering, vol. 22, pp. 1345-1359, 2010.

[16] M. Kaboli, "Leveraging over prior knowledge for online learning of visual categories across robots.," Thesis, KTH, 2012.

[17] Y. Zhan and M. E. Taylor, "Online transfer learning in reinforcement learning domains.," arXiv:1507.00436, 2015.

[18] M. Kaboli, P. Mittendorfer, V. Hugel, and G. Cheng, "Humanoids learn object properties from robust tactile feature descriptors via multimodal artificial skin," IEEE-RAS Int. Conf. on Humanoid Robots, pp. 187-192, 2014.

[19] M. Kaboli, A. DeLaRosaT, R. Walker, and G. Cheng, "In-hand object recognition via texture properties with robotic hands, artificial skin, and novel tactile descriptors," IEEE-RAS Int. Conf. on Humanoid Robots, pp. 1155-1160, 2015.

[20] M. Kaboli, A. Long, and G. Cheng, "Humanoids learn touch modalities identification via multi-modal robotic skin and robust tactile descriptors," Advanced Robotics, vol. 29, no. 21, pp. 1411-1425, 2015.

[21] T. Tommasi, F. Orabona, M. Kaboli, and B. Caputo, "Leveraging over prior knowledge for online learning of visual categories," British Machine Vision Conference, 2012.

[22] J. A. K. Suykens and J. Vandewalle, "Least squares support vector machine classifiers," Neural Process. Lett., vol. 9, pp. 293-300, 1999.

[23] K. Crammer, O. Dekel, J. Keshet, S. Shalev-Shwartz, and Y. Singer, "Online passive-aggressive algorithms," J. of Machine Learning Research, vol. 7, pp. 551-585, 2006. 